\title{
Solvent-free highly regioselective Michael addition of phenytoin to $\alpha, \beta$-unsaturated esters in the presence of TBAB under ultrasound irradiation
}

\author{
Gholamhassan Imanzadeh* and Somayeh Rezaee-Gatar \\ Department of Chemistry, Faculty of Basic Science, University of Mohaghegh Ardabili \\ 56199-11367 Ardabil, Iran \\ E-mail: imanzad2000@yahoo.com
}

DOI:http://dx.doi.org/10.3998/ark.5550190.p009.048

\begin{abstract}
The highly regioselective $\mathrm{C}-\mathrm{N}$ bond formation between phenytoin and acrylic/fumaric esters was accomplished. Solvent-free media Michael addition of phenytoin to acrylic esters led to the selective synthesis of $\mathrm{N3}$-substituted phenytoins (as mono-Michael adducts) in the presence of tetrabutylammonium bromide $(\mathrm{TBAB})$ and potassium carbonate under ultrasonic irradiation conditions at room temperature. These reactions produced N1,N3-disubstituted phenytoins (as bis-Michael adducts) at $70{ }^{\circ} \mathrm{C}$. Surprisingly, the addition of phenytoin to fumaric esters, instead of acrylic esters, selectively provided only $\mathrm{N3}$-substituted phenytoins at both room temperature and $70^{\circ} \mathrm{C}$ under the same conditions.
\end{abstract}

Keywords: Solvent-free, regioselective, Michael addition, phenytoin, $\alpha, \beta$-unsaturated esters, ultrasound irradiation

\section{Introduction}

Among the five-membered ring compounds with two heteroatoms, hydantoin has high importance due to key building blocks for a large number of medicinally-relevant molecules and serves as fundamental scaffold for discovering drugs. ${ }^{1}$ The chemistry and property of hydantoin and its derivatives have been investigated for more than 140 years. These studies indicate that hydantoin and its derivatives have a broad range of biological activities including anticonvulsants, ${ }^{2}$ antiarithmics, ${ }^{3}$ antitumor, ${ }^{4}$ antidepressants, ${ }^{5}$ and antiviral agents. ${ }^{6}$ Phenytoin 1 (5,5-diphenylhydantoin, DPH) is one of the best known derivatives of hydantoin. This heterocycle is a useful pharmacological compound and extensively used as an anticonvulsant agent, ${ }^{7}$ treatment of epileptic seizures, ${ }^{8,9}$ epidermolysis bullosa, ${ }^{10}$ inflammatory conditions, ${ }^{11}$ cardiac antiarrhythmic, ${ }^{12}$ and for the treatment of many more diseases including HIV. ${ }^{13}$ In the 
case of anticonvulsant activity of DPH, it has been established that the site of the action of this compound is the neuronal voltage-sensitive sodium channel, though no more information about the location and nature of this site has been reported. ${ }^{14,15}$

In contrast to the many examples of the Michael addition amines on different unsaturated systems, there are only a few reports on the amides and imides Michael reaction (due to the low reactivity of them) with these systems. ${ }^{16-19}$ Although, neutral amides and imides have very restricted nucleophilicity, but in the presence of a convenient base, nitrogen anions derived from them can become more satisfactory nucleophiles in Michael-type reactions. ${ }^{20}$ DPH contains both amide and imide moieties. Therefore, it contains two Michael donor positions, which under the treatment of proper base, may generate $\mathrm{Nl}$-position or N3-position DPH anions. Therefore, it is expected to have three possible products from reaction of DPH with an electrophile in the base media (Scheme 1).

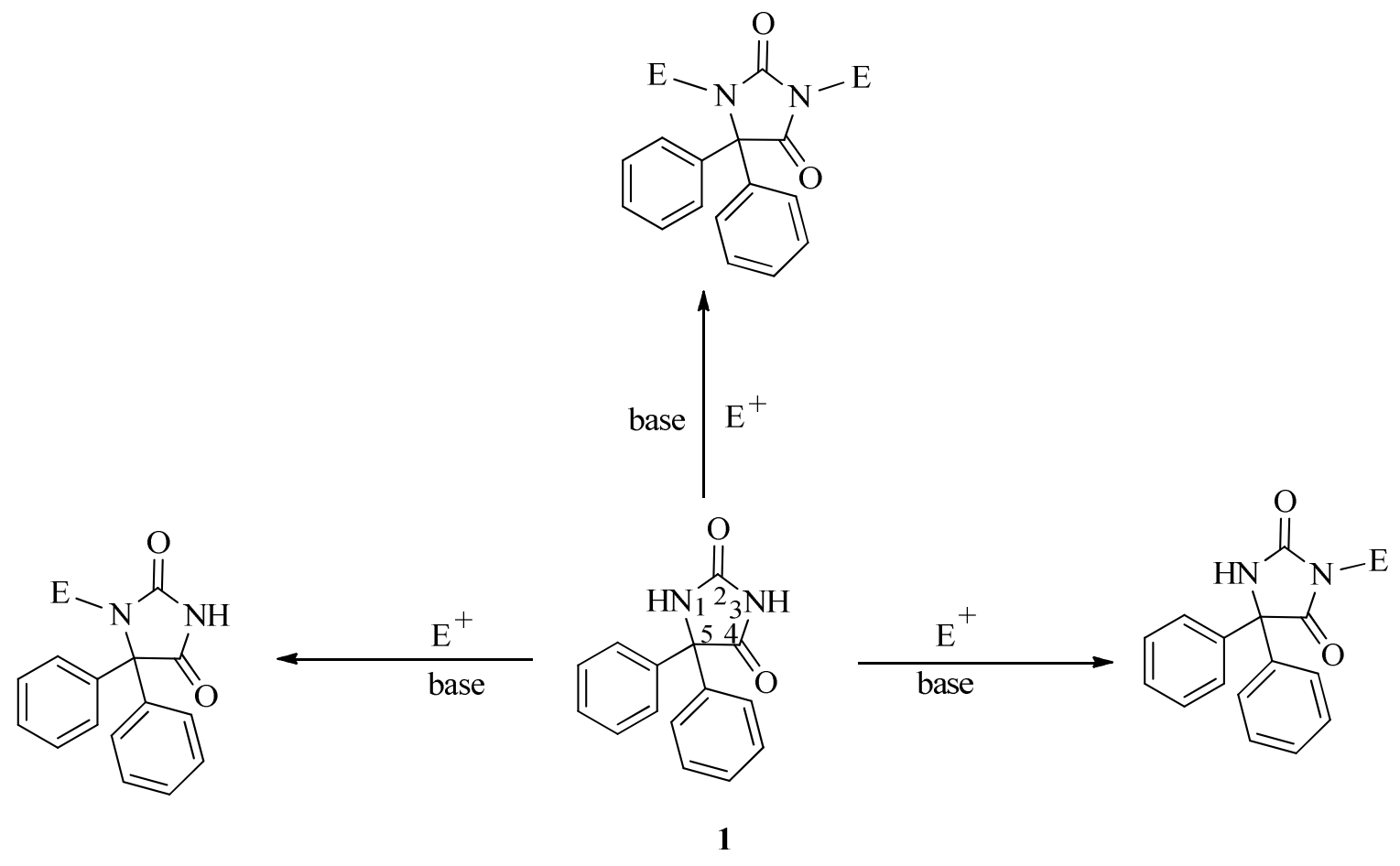

Scheme 1. Nucleophilic reactions of diverse positions of DPH with an electrophile.

Ultrasound is a powerful tool that can be used in organic synthesis to achieve a number of chemical reactions in high yield and within a shorter reaction time. Therefore, recently, ultrasonic irradiation technique has received considerable attention of researchers. ${ }^{21-25}$ In continuation of our ongoing research on the Michael addition of imides and amides to unsaturated systems, ${ }^{26,27}$ we report here an efficient strategy for synthesis of a series of DPH derivatives by Michael addition of this compound to $\alpha, \beta$-unsaturated esters under ultrasound irradiation (Scheme 2). 


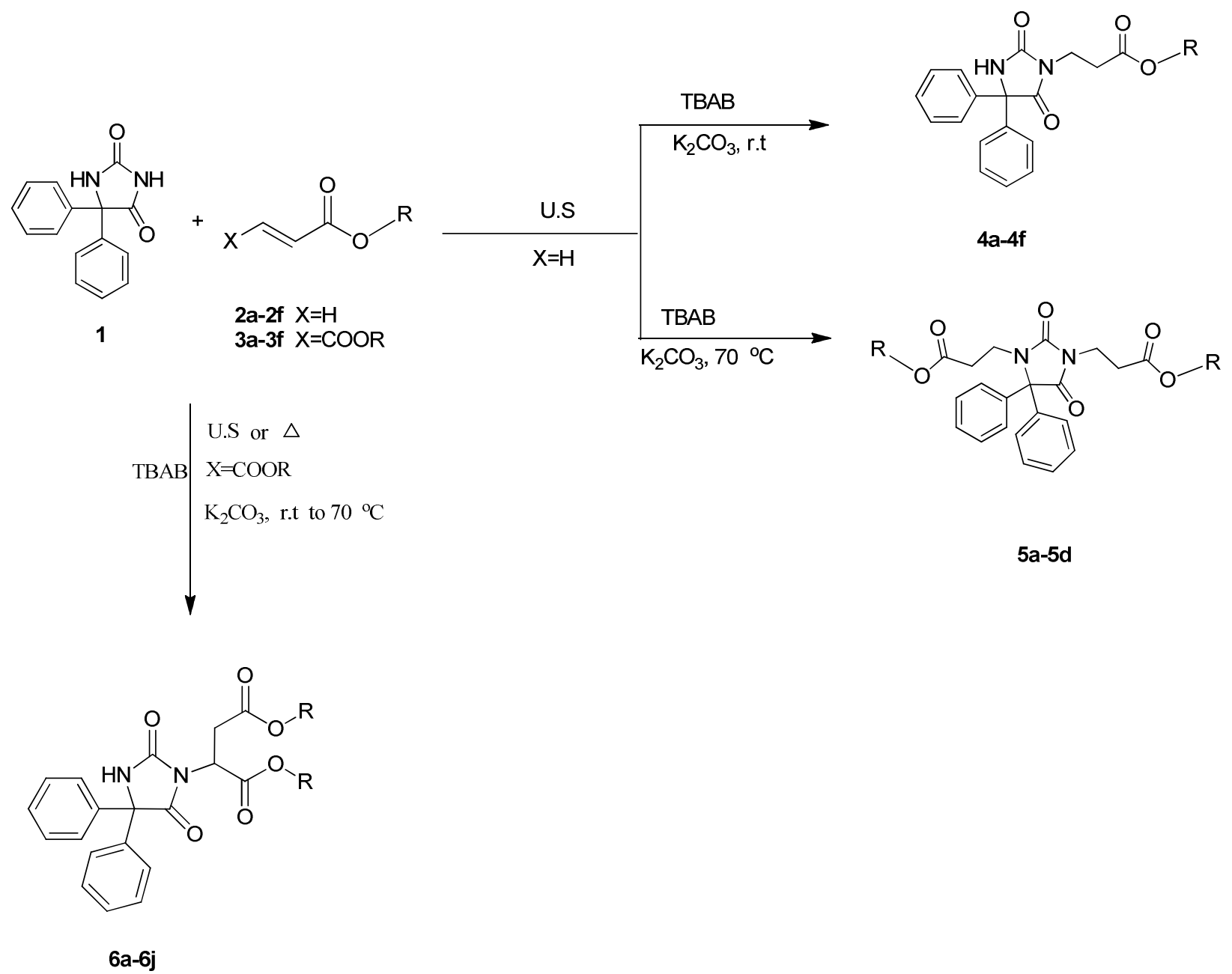

Scheme 2. Michael addition of DPH to $\alpha, \beta$-unsaturated esters under both conventional heating and ultrasound irradiation.

\section{Results and Discussion}

The general method for the preparation of DPH involves the base catalysed condensation of benzil and urea, known as the Biltz synthesis of $\mathrm{DPH} .{ }^{28}$ Using this reaction, we prepared the starting material in $90 \%$ yield under ultrasound irradiation. Subsequently, we performed a model Michael addition reaction in which $n$-butyl acrylate $(\mathrm{R}=n$ - $\mathrm{Bu}, \mathrm{X}=\mathrm{H}, 1 \mathrm{mmol})$ was used as a Michael acceptor substrate to react with DPH $(1 \mathrm{mmol})$ in the presence of TBAB, as a strong ionic salt, at room temperature under solvent-free and ultrasound irradiation conditions. Initially, this model reaction was examined in five different organic and inorganic bases $(1 \mathrm{mmol})$, pyridine, triethylamine, 1,4-diaza-bicyclo[2,2,2]octane (DABCO), sodium carbonate and potassium carbonate. Surprisingly, we found that the reaction took place regioselectively at the N3-position of DPH and produced the corresponding mono-Michael adduct 4d as the sole 
product in the presence of each of these bases. Among these bases, potassium carbonate gave the best result under the ultrasound irradiation. We repeated the model reaction under the above conditions in the presence of $2 \mathrm{mmol}$ potassium carbonate and $2 \mathrm{mmol}$ butyl acrylate that after irradiation of the reaction mixture for $2 \mathrm{~h}$, the mono-adduct $\mathbf{4 d}$, based on TLC test, was the only afforded product and no bis-adduct $\mathbf{5 b}$ was obtained at all. Interestingly, by increasing the temperature of reaction mixture to $70{ }^{\circ} \mathrm{C}$, bis-Michael adduct $\mathbf{5 b}$ was obtained as sole product after $1 \mathrm{~h}$ irradiation (Scheme 3 ). The molar ratio of base was also optimized, and a good yield was obtained by employing 1 equiv of potassium carbonate.

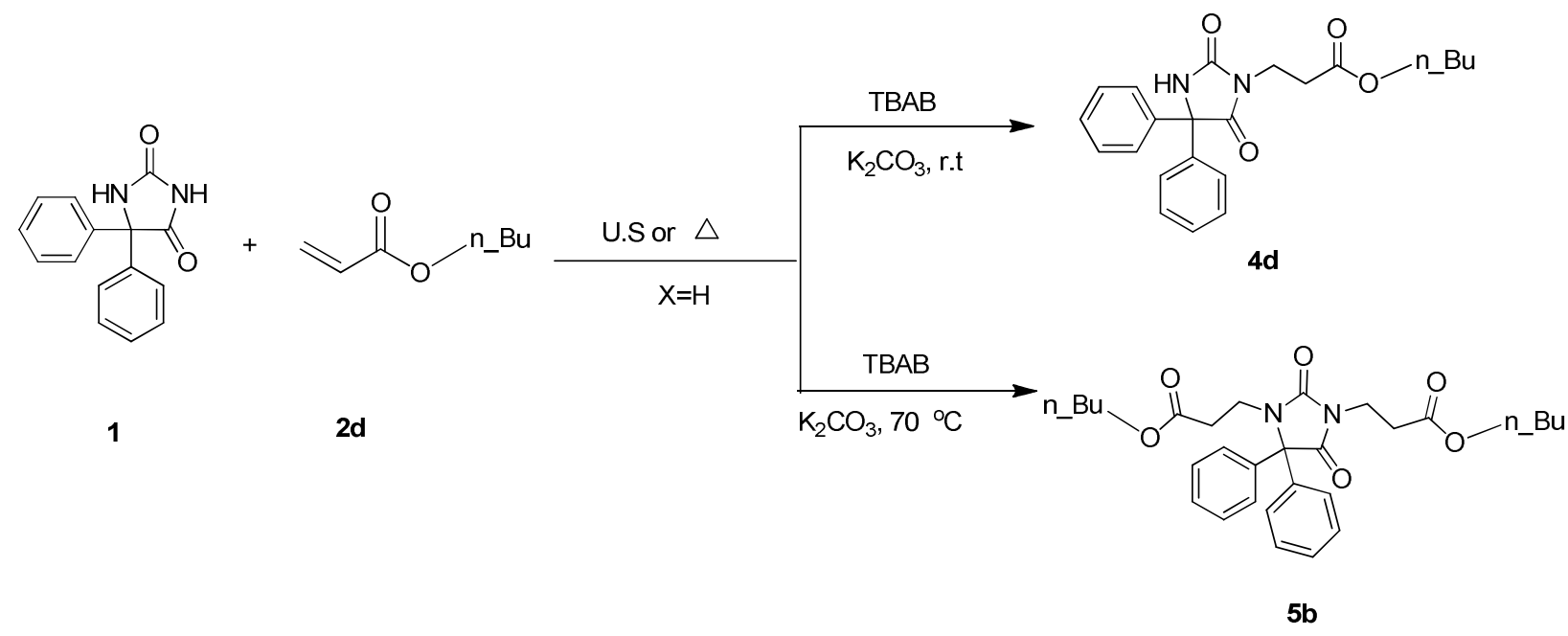

Scheme 3. Michael addition of DPH to butyl acrylate under both conventional heating and ultrasound irradiation.

To evaluate the effects of solvents and ultrasound irradiation on the reactivity of reagents, the model reaction was performed in diverse solvents under both conventional heating and ultrasound irradiation conditions (Table 1). The best yields of products were obtained when $\mathrm{TBAB}$ was used as a strong ionic salt media under ultrasound irradiation (Table 1, entry 6). The desired mono-adduct $4 \mathbf{d}$ was isolated in $80 \%$ yield within $2 \mathrm{~h}$ in presence of TBAB and under ultrasound irradiation (Table 1, entry 6). The reaction, within the same time, produced $30 \%$ yield of the Michael adduct $\mathbf{4 d}$ when it was performed under conventional heating condition (Table 1, entry 6). Although, moderate yields of mono-adduct $4 \mathbf{d}$ were afforded in DMF and DMSO solvents, bis-adducts $\mathbf{5 b}$ were obtained in very low yields in these solvents (Table 1, entries 1 and 2). The reaction was unsuccessful in water as well as under solvent-free conditions (Table 1, entries 5 and 7). Ionic organic salts are solvents with high polarity and consequently capable to dissolve organic compounds and metallic salts. ${ }^{29}$ Therefore, in our reaction, we can think that organic salt $\mathrm{TBAB}$ play role of a high polar solvent and accelerates the reaction with dissolving all of organic (ester and $\mathrm{DPH})$ and inorganic salt $\left(\mathrm{K}_{2} \mathrm{CO}_{3}\right)$ reactants. 
Table 1. Effect of solvents on regioselective aza-Michael reaction of $n$-butyl acrylate and phenytoin

\begin{tabular}{cccccc}
\hline \multirow{2}{*}{ Entry } & \multirow{2}{*}{ Solvent } & \multicolumn{2}{c}{ Time h/(Yield $\left.\mathbf{4 d} \%^{\mathrm{a}}\right)^{\mathrm{b}}$} & \multicolumn{2}{c}{ Time h/(Yield $\left.\left.\mathbf{5} \mathbf{b}^{\mathrm{a}}\right)^{\mathrm{c}}\right)^{\mathrm{c}}$} \\
\cline { 3 - 6 } & & $\mathrm{US}$ & $\Delta$ & $\mathrm{US}$ & $\Delta$ \\
\hline 1 & $\mathrm{DMF}$ & $2 /(30)$ & $2 /(30)$ & $8 /(5)$ & $8 /(5)$ \\
2 & $\mathrm{DMSO}$ & $2 /(50)$ & $2 /(10)$ & $8 /(5)$ & $8 /(0)$ \\
3 & $\mathrm{EtOH}$ & $2 /(20)$ & $2 /(5)$ & $8 /(10)$ & $8 /(10)$ \\
4 & $\mathrm{CH}_{3} \mathrm{COCH}$ & $2 /(10)$ & $2 /(10)$ & $8 /(0)$ & $8 /(0)$ \\
5 & $\mathrm{H}_{2} \mathrm{O}$ & $2 /(0)$ & $2 /(0)$ & $8 /(0)$ & $8 /(0)$ \\
6 & $\mathrm{TBAB}$ & $2 /(80)$ & $2 /(30)$ & $8 /(50)$ & $8 /(20)$ \\
7 & No solvent & $2 /(0)$ & $2 /(0)$ & $8 /(0)$ & $8 /(0)$ \\
\hline
\end{tabular}

a Isolated yield.

b The reactions were performed with phenytoin $(1 \mathrm{mmol})$, potassium carbonate $(1 \mathrm{mmol})$ and $n$ butylacrylate $(1 \mathrm{mmol})$ in tetrabutylammonium bromide $(1 \mathrm{mmol}) 5 \mathrm{~mL}$ solvent at room temperature.

${ }^{\mathrm{c}}$ The reactions were performed with phenytoin $(1 \mathrm{mmol})$, potassium carbonate $(1 \mathrm{mmol})$ and $n$ butylacrylate $(2 \mathrm{mmol})$ in tetrabutylammonium bromide $(1 \mathrm{mmol})$ or $5 \mathrm{~mL}$ solvent at $70{ }^{\circ} \mathrm{C}$.

Encouraged by this highly regioselectve result, the model reaction was repeated with butyl fumarate, instead of butyl acrylate (Scheme 4). We observed a surprising phenomenon that the reaction provided mono-Michael adduct $\mathbf{6 c}$ as the only product at both room temperature and 70 ${ }^{\circ} \mathrm{C}$ when the butyl fumarate and potassium carbonate loadings were changed from 1 equiv to 2 equiv (Scheme 4). We postulated that butyl fumarate can be considered as a derivatives of butyl acrylate, therefore, the presence of a butyloxycarbonyl (-COOn-Bu) in the $\beta$-position of n-butyl acrylate prevents the attacking of high hindrance nucleophilic N1-position, due to the steric effect of the two phenyl groups at $C 5$ in DPH.

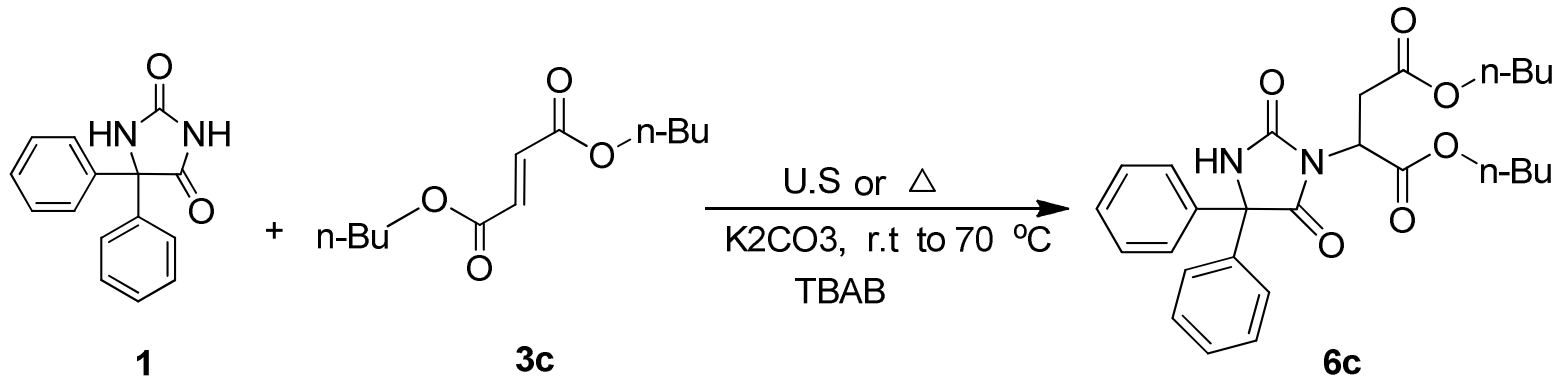

Scheme 4. Michael addition of DPH to n-butyl fumarate under both conventional heating and ultrasound irradiation. 
Table 2. Regioselective Michael addition of DPH to acrylic and fumaric esters under ultrasound irradiation and conventional thermal heating conditions

\begin{tabular}{|c|c|c|c|c|c|c|c|c|}
\hline \multirow{2}{*}{ Entry } & \multirow{2}{*}{$\mathrm{X}$} & \multirow{2}{*}{$\mathrm{R}$} & \multirow{2}{*}{ Ester } & \multirow{2}{*}{ Product } & \multicolumn{2}{|c|}{ Time $(\mathrm{h})$} & \multicolumn{2}{|c|}{ Yield $(\%)^{\mathrm{c}}$} \\
\hline & & & & & U.S & $\Delta$ & U.S & $\Delta$ \\
\hline $1^{\mathrm{a}}$ & $\mathrm{H}$ & $\mathrm{CH}_{3}$ & $2 \mathbf{a}$ & $4 \mathbf{a}$ & 72 & 10 & - & - \\
\hline $2^{\mathrm{a}}$ & $\mathrm{H}$ & $\mathrm{CH}_{3} \mathrm{CHCH}_{3}$ & $2 \mathbf{b}$ & $4 b$ & 72 & 10 & - & - \\
\hline $3^{\mathrm{a}}$ & $\mathrm{H}$ & $\mathrm{CH}_{3} \mathrm{CH}_{2}$ & $2 c$ & $4 c$ & 2 & 1 & 93 & 96 \\
\hline $4^{\mathrm{a}}$ & $\mathrm{H}$ & $\mathrm{CH}_{3}\left(\mathrm{CH}_{2}\right)_{3}$ & 2d & 4d & 4 & 2 & 85 & 89 \\
\hline $5^{\mathrm{a}}$ & $\mathrm{H}$ & $\mathrm{CH}_{3}\left(\mathrm{CH}_{2}\right)_{5}$ & $2 e$ & $4 e$ & 7 & 2 & 80 & 83 \\
\hline $6^{\mathrm{a}}$ & $\mathrm{H}$ & $\mathrm{CH}_{3}\left(\mathrm{CH}_{2}\right)_{3} \mathrm{CH}\left(\mathrm{CH}_{3} \mathrm{CH}_{2}\right) \mathrm{CH}_{2}$ & $2 f$ & $4 f$ & 10 & 3.5 & 76 & 71 \\
\hline $7^{\mathrm{b}}$ & $\mathrm{H}$ & $\mathrm{CH}_{3} \mathrm{CH}_{2}$ & $2 c$ & $5 \mathbf{a}$ & 3 & 1.5 & 75 & 85 \\
\hline $8^{\mathrm{b}}$ & $\mathrm{H}$ & $\mathrm{CH}_{3}\left(\mathrm{CH}_{2}\right)_{3}$ & 2d & $5 \mathbf{b}$ & 8 & 2 & 76 & 83 \\
\hline $9^{\mathrm{b}}$ & $\mathrm{H}$ & $\mathrm{CH}_{3}\left(\mathrm{CH}_{2}\right)_{5}$ & $2 e$ & $5 c$ & 10 & 3 & 72 & 80 \\
\hline $10^{\mathrm{b}}$ & $\mathrm{H}$ & $\mathrm{CH}_{3}\left(\mathrm{CH}_{2}\right)_{3} \mathrm{CH}\left(\mathrm{CH}_{3} \mathrm{CH}_{2}\right) \mathrm{CH}_{2}$ & $2 f$ & 5d & 19 & 5 & 60 & 65 \\
\hline $11^{\mathrm{c}}$ & COOR & $\mathrm{CH}_{3} \mathrm{CH}_{2}$ & 3a & $\mathbf{6 a}$ & 36 & 8 & 63 & 74 \\
\hline $12^{\mathrm{c}}$ & COOR & $\mathrm{CH}_{3} \mathrm{CH}_{2} \mathrm{CH}_{2}$ & $\mathbf{3 b}$ & $6 \mathbf{b}$ & 40 & 9 & 61 & 72 \\
\hline $13^{\mathrm{c}}$ & COOR & $\mathrm{CH}_{3} \mathrm{CH}_{2} \mathrm{CH}_{2} \mathrm{CH}_{2}$ & $3 c$ & 6c & 48 & 9 & 55 & 69 \\
\hline $14^{\mathrm{c}}$ & COOR & $\mathrm{CH}_{3}\left(\mathrm{CH}_{2}\right)_{4}$ & 3d & 6d & 50 & 9 & 52 & 67 \\
\hline $15^{\mathrm{c}}$ & COOR & $\mathrm{CH}_{3}\left(\mathrm{CH}_{2}\right)_{7}$ & $3 e$ & $6 e$ & 40 & 11 & 49 & 64 \\
\hline $16^{\mathrm{c}}$ & COOR & $\mathrm{CH}_{3}\left(\mathrm{CH}_{2}\right)_{9}$ & 3f & $6 \mathbf{f}$ & 52 & 15 & 41 & 60 \\
\hline $17^{\mathrm{c}}$ & COOR & $\mathrm{CH}_{3} \mathrm{CHCH}_{3}$ & $3 g$ & $6 \mathrm{~g}$ & 72 & 10 & - & - \\
\hline $18^{\mathrm{c}}$ & COOR & $\mathrm{CH}_{3} \mathrm{CH}\left(\mathrm{CH}_{2}\right)_{2} \mathrm{CH}_{3}$ & $3 \mathbf{h}$ & $6 h$ & 72 & 10 & - & - \\
\hline $19^{c}$ & COOR & cyclohexyl & $3 \mathbf{i}$ & $6 \mathbf{i}$ & 72 & 10 & - & - \\
\hline $20^{\mathrm{c}}$ & COOR & $\mathrm{Ph}$ & $\mathbf{3 j}$ & $6 \mathbf{j}$ & 72 & 10 & - & - \\
\hline
\end{tabular}

${ }^{\text {a }}$ The reaction was performed with DPH $(1 \mathrm{mmol})$, TBAB $(1 \mathrm{mmol})$, potassium carbonate $(1$ mmol) and acrylic ester $(1 \mathrm{mmol})$ at room temperature; ${ }^{\mathrm{b}}$ The reaction was performed with DPH $(1 \mathrm{mmol})$, TBAB $(1 \mathrm{mmol})$, potassium carbonate $(1 \mathrm{mmol})$ and acrylic ester $(2 \mathrm{mmol})$ at $70{ }^{\circ} \mathrm{C} ;{ }^{\mathrm{c}}$ The reaction was performed with DPH $(1 \mathrm{mmol})$, TBAB $(1 \mathrm{mmol})$, potassium carbonate $(1$ $\mathrm{mmol})$ and fumaric ester $(1 \mathrm{mmol})$ at room temperature; ${ }^{\mathrm{d}}$ Isolated yield.

Under the optimized conditions (see footnotes $b$ and $c$ of Table 1), other acrylic and fumaric esters were examined to explore the scope and limitations of this method and the results were summarized in Table 2. Upon the analysis of Table 2, it could be verified that the reaction could produce the desired mono-Michael adducts, as the only regioisomers, and bis-Michael adducts at room temperature and $70{ }^{\circ} \mathrm{C}$ respectively in good to high yields under both fore-mentioned conditions. However, compared with conventional heating conditions, most of reaction products have higher yields and the related reaction times are significantly lower under ultrasound 
irradiation conditions (Table 2, entries 3-16). Long chain alkoxy groups like n-octyloxy, ndecyloxy of fumaric esters were less reactive under the ultrasound irradiation conditions (Table 2 , entries 15 and 16). The reaction was not successful with acrylic or fumaric esters that bear phenoxy, secondary and tertiary alkoxy groups (Table 2, entries 2 and 17-20).

When methyl acrylate was employed as Michael acceptor under thermal heating or ultrasound irradiation conditions, acrylic acid and DPH 1 were obtained instead of the desired Michael adduct (Table 2, entry 1). We believe that methyl fumarate is more susceptible to hydrolysis, due to its smaller alkoxy group (-OMe), under the reaction conditions.

\section{Conclusions}

In conclusion, solvent-free ultrasound-induced high regioselective Michael addition, in the presence of TBAB, of DPH to $\alpha, \beta$-unsaturated esters was reported. We have found that at room temperature the Michael-addition reaction between DPH and acrylic esters lead exclusively to $N 3$-substituted phenytoins under ultrasound irradiation in solvent-free media. While, at $70{ }^{\circ} \mathrm{C}$, the reaction products were $N 1, N 3$-disubstituted phenytoins. When acrylic esters were replaced by fumaric esters, only $\mathrm{N3}$-substituted phenytoins were obtained as Michael adducts at both room temperature and $70{ }^{\circ} \mathrm{C}$. This remarkable regioselectivity can be related to high steric hindrance of phenyl groups at C5-position that reduce the nucleophilicity of the N1-position of DPH.

\section{Experimental Section}

General. All acrylic and fumaric esters were synthesized in our laboratory according to the literature procedure ${ }^{30}$ and their structures were confirmed by IR and ${ }^{1} \mathrm{H}$ NMR spectroscopy. The progress of the reactions was followed by TLC using silica gel SILIG/UV 254 plates. ${ }^{1} \mathrm{H}$ NMR $(400 \mathrm{MHz})$ and ${ }^{13} \mathrm{C}$ NMR $(100 \mathrm{MHz})$ spectra were recorded on a Bruker $400 \mathrm{MHz}$ instrument. FT-IR spectra were recorded on a Perkin-Elmer RX-1 instrument. Elemental analysis for C, H, and $\mathrm{N}$ were performed using a Heraeus $\mathrm{CHN}-\mathrm{O}-$ Rapid analyzer. The melting points were determined in open capillaries with a Stuart Melting Point Apparatus and are uncorrected. Sonication was performed in a Parsonic 7500s ultrasonic bath, with a frequency of $50 \mathrm{kHz}$ and a power $100 \mathrm{~W}$ was used for ultrasonic irradiation.

\section{General procedure under conventional conditions (method $A$ )}

$\alpha, \beta$-Unsaturated ester $(2 \mathrm{mmol})$ was added to a well ground mixture of DPH ( $2 \mathrm{mmol})$, TBAB (2 $\mathrm{mmol})$ and $\mathrm{K}_{2} \mathrm{CO}_{3}(2 \mathrm{mmol})$ and mixed thoroughly with a glass rod. The resulting mixture was kept at room temperature, or in an oil bath at $70{ }^{\circ} \mathrm{C}$ for appropriate time (Table 2). The progress of reaction was monitored by TLC. After completion of reaction, the mixture was suspended in chloroform $(40 \mathrm{~mL})$, filtered and the filtrate was washed with water $(3 \times 20 \mathrm{ml})$ and dried with 
$\mathrm{MgSO}_{4}$. The solvent was removed under reduced pressure and the resulting crude material was purified on short silica-gel column with $n$-hexane/ethyl acetate (9.5:0.5) as the eluent.

\section{General procedure under sonochemical conditions (method B)}

$\alpha, \beta$-Unsaturated ester $(2 \mathrm{mmol})$ was added to a well ground mixture of DPH (2 mmol), TBAB (2 $\mathrm{mmol})$ and $\mathrm{K}_{2} \mathrm{CO}_{3}(2 \mathrm{mmol})$ and mixed thoroughly with a glass rod. The mixture was irradiated in the water bath of an ultrasonic cleaner at $25{ }^{\circ} \mathrm{C}$ or $70{ }^{\circ} \mathrm{C}$. The progress of reaction was monitored by TLC. After completion of reaction, the mixture was suspended in chloroform (40 $\mathrm{mL})$, filtered and the filtrate was washed with water $(3 \times 20 \mathrm{ml})$ and dried with $\mathrm{MgSO}_{4}$. The solvent was removed under reduced pressure and the resulting crude material was purified on short silica-gel column with $n$-hexane/ethyl acetate (9.5:0.5) as the eluent.

Ethyl 3-(2,5-dioxo-4,4diphenylimidazolidin-1-yl)propanoate (4c). White solid, mp96-98 ${ }^{\circ} \mathrm{C}$. FT-IR ( $\left.v_{\max }, \mathrm{cm}^{-1}\right): 3243,3091,2984,1769,1733,1706,1448,1195,709 .{ }^{1} \mathrm{H}$ NMR (400 MHz, $\left.\mathrm{CDCl}_{3}\right): \delta_{\mathrm{H}} 1.18\left(\mathrm{t}, 3 \mathrm{H}, J 7.2 \mathrm{~Hz}, \mathrm{CH}_{3}\right), 2.7\left(\mathrm{t}, 2 \mathrm{H}, J 7.2 \mathrm{~Hz}, \mathrm{CH}_{2}\right), 3.9\left(\mathrm{t}, 2 \mathrm{H}, J 7.2 \mathrm{~Hz}, \mathrm{CH}_{2}\right), 4.1$ (q, $2 \mathrm{H}, J$ 7.2 Hz, $\mathrm{CH}_{2}$ ), 7.29-7.41 (m, 11H, NH, Ar). ${ }^{13} \mathrm{C} \mathrm{NMR}\left(100 \mathrm{MHz}, \mathrm{CDCl}_{3}\right): \delta_{\mathrm{C}} 14.06$, 32.62 , 34.92, 60.88, 70.13, 126.9, 128.6, 128.82, 139.03, 156.42, 170.64, 173.08. Anal. calcd for $\mathrm{C}_{20} \mathrm{H}_{20} \mathrm{~N}_{2} \mathrm{O}_{4}$ : C, 68.17; H, 5.72; N, 7.95. Found: C, 68.21; H, 5.83; N, 7.91.

Butyl 3-(2,5-dioxo-4,4-diphenylimidazolidin-1-yl)propanoate (4d). White solid, mp91-93 ${ }^{\circ} \mathrm{C}$. FT- IR $\left(v_{\max }, \mathrm{cm}^{-1}\right): 3283,2959,1774,1747,1706,1452,1173,720 .{ }^{1} \mathrm{H}$ NMR (400 MHz, $\mathrm{CDCl}_{3}$ ): $\delta_{\mathrm{H}} 0.91\left(\mathrm{t}, 3 \mathrm{H}, J 7.6 \mathrm{~Hz}, \mathrm{CH}_{3}\right.$ ), 1.33 (sxt, $2 \mathrm{H}, J 7.6 \mathrm{~Hz}, \mathrm{CH}_{2}$ ), 1.54 (quint, $2 \mathrm{H}, J 6.8 \mathrm{~Hz}$, $\mathrm{CH}_{2}$ ), $2.71\left(\mathrm{t}, 2 \mathrm{H}, J 7.6 \mathrm{~Hz}, \mathrm{CH}_{2}\right), 3.88\left(\mathrm{t}, 2 \mathrm{H}, J 7.6 \mathrm{~Hz}, \mathrm{CH}_{2}\right), 4.03\left(\mathrm{t}, 2 \mathrm{H}, J 6.8 \mathrm{~Hz}, \mathrm{CH}_{2}\right), 7.29-$ $7.39(\mathrm{~m}, 11 \mathrm{H}, \mathrm{NH}, \mathrm{Ar}) .{ }^{13} \mathrm{C} \mathrm{NMR}\left(100 \mathrm{MHz}, \mathrm{CDCl}_{3}\right): \delta_{\mathrm{C}} 13.71,19.07,30.47,32.56,34.88$, $64.79,70.12,126.88,128.59,128.82,139.04,156.41,170.70,173.07$. Anal. calcd for $\mathrm{C}_{22} \mathrm{H}_{24} \mathrm{~N}_{2} \mathrm{O}_{4}$ : C, 69.46; H, 6.36; N, 7.36. Found: C, 69.52; H, 6.38; N, 7.42.

Hexyl 3-(2,5-dioxo-4,4-diphenylimidazolidin-1-yl)propanoate (4e). White solid, mp82-85 ${ }^{\circ} \mathrm{C}$. FT- IR $\left(v_{\max }, \mathrm{cm}^{-1}\right): 3288,2959,2932,1743,1772,1704,1452,1178,763,699,606 .{ }^{1} \mathrm{H}$ NMR $\left(400 \mathrm{MHz}, \mathrm{CDCl}_{3}\right): \delta_{\mathrm{H}} 0,91\left(\mathrm{t}, 3 \mathrm{H}, J 7.2 \mathrm{~Hz}, \mathrm{CH}_{3}\right), 1.29-1.34\left(\mathrm{~m}, 6 \mathrm{H}, 3 \mathrm{CH}_{2}\right), 1.56$ (quint, $2 \mathrm{H}, J$ $7.2 \mathrm{~Hz}, \mathrm{CH}_{2}$ ), 2.73 (t, $\left.2 \mathrm{H}, J 7.2 \mathrm{~Hz}, \mathrm{CH}_{2}\right), 3.9$ (t, $\left.2 \mathrm{H}, J 7.2 \mathrm{~Hz}, \mathrm{CH}_{2}\right), 4.03$ (t, 2H, J 7.2 Hz, $\mathrm{CH}_{2}$ ), 6.44 (br, $1 \mathrm{H}, \mathrm{NH}), 7.29-7.42$ (m, 10H, Ar). ${ }^{13} \mathrm{C} \mathrm{NMR}\left(100 \mathrm{MHz}, \mathrm{CDCl}_{3}\right): \delta_{\mathrm{C}} 14.03,22.54,25.51$, 28.42 , 31.42, 23.54, 34.94, 65.10, 70.12, 126.91, 128.86, 138.98, 170.67, 172.99. Anal. calcd for $\mathrm{C}_{24} \mathrm{H}_{28} \mathrm{~N}_{2} \mathrm{O}_{4}$ : C, 70.57; H, 6.91; N, 6.86. Found: C, 70.51; H, 6.88; N, 6.86.

2-Ethylhexyl 3-(2,5-dioxo-4,4-diphenylimidazolidin-1-yl)propanoate (4f). Colourless oil. FTIR $\left(v_{\max }, \mathrm{cm}^{-1}\right): 3293,2961,2932,1772,1744,1708,1449,1188,707,603,492 .{ }^{1} \mathrm{H}$ NMR (400 $\mathrm{MHz}_{\mathrm{CDCl}}$ ): $\delta_{\mathrm{H}} 0.88\left(\mathrm{t}, 3 \mathrm{H}, J 7.2 \mathrm{~Hz}, \mathrm{CH}_{3}\right), 0.91\left(\mathrm{t}, 3 \mathrm{H}, J 6.8 \mathrm{~Hz}, \mathrm{CH}_{3}\right), 1.28-1.39(\mathrm{~m}, 8 \mathrm{H}$, $\left.4 \mathrm{CH}_{2}\right), 1.52-1.56(\mathrm{~m}, 1 \mathrm{H}, \mathrm{CH}), 2.71\left(\mathrm{t}, 2 \mathrm{H}, J 7.6 \mathrm{~Hz}, \mathrm{CH}_{2}\right), 3.88\left(\mathrm{t}, 2 \mathrm{H}, J 7.6 \mathrm{~Hz}, \mathrm{CH}_{2}\right), 3.93-$ $4.02\left(\mathrm{~m}, 2 \mathrm{H}, \mathrm{CH}_{2}\right), 7.29-7.43$ (m, 10H, Ar), 7.88 (s, br, 1H, NH). ${ }^{13} \mathrm{C} \mathrm{NMR}\left(\mathrm{CDCl}_{3}, \mathrm{TMS}, 100\right.$ $\mathrm{MHz}): \delta_{\mathrm{C}} 10.95,14.10,22.99,23.66,28.88,30.28,32.52,34.79,38.59,67.34,70.13,126.87$, 128.54, 128.80, 139.09, 170.80, 173.14. Anal. calcd for $\mathrm{C}_{26} \mathrm{H}_{32} \mathrm{~N}_{2} \mathrm{O}_{4}$ : C, 71.53; H, 7.39; N, 6.42. Found: C, 71.51; H, 7.43; N, 6.38 . 
Diethyl 1,3-di-(2,5-dioxo-4,4-diphenylimidazolidin-1-yl)propanoate (5a). White Solid, mp83$85^{\circ} \mathrm{C}$. FT-IR $\left(v_{\max }, \mathrm{cm}^{-1}\right): 2984,2933,1771,1729,1717,1455,1197,1020,719 .{ }^{1} \mathrm{H}$ NMR $(400$ $\left.\mathrm{MHz}, \mathrm{CDCl}_{3}\right): \delta_{\mathrm{H}} 1.18\left(\mathrm{t}, 3 \mathrm{H}, J 7.2 \mathrm{~Hz}, \mathrm{CH}_{3}\right), 1.22\left(\mathrm{t}, 3 \mathrm{H}, J 7.2 \mathrm{~Hz}, \mathrm{CH}_{3}\right), 1.93\left(\mathrm{~m}, 2 \mathrm{H}, \mathrm{CH}_{2}\right)$, $2.73\left(\mathrm{t}, 2 \mathrm{H}, J 7.2 \mathrm{~Hz}, \mathrm{CH}_{2}\right), 3.66-3.70\left(\mathrm{~m}, 2 \mathrm{H}, \mathrm{CH}_{2}\right), 3.90\left(\mathrm{t}, 2 \mathrm{H}, J 7.2 \mathrm{~Hz}, \mathrm{CH}_{2}\right), 4.02$ (q, 2H, $\left.7.2 \mathrm{~Hz}, \mathrm{CH}_{2}\right), 4.10$ (q, 2H, J 7.2 Hz, $\left.\mathrm{CH}_{2}\right), 7.28-7.44$ (m, 10H, Ar). ${ }^{13} \mathrm{C}$ NMR (100 MHz, CDCl $)_{3}$ : $\delta_{\mathrm{C}} 14.08,14.11,32.31,32.68,35.24,37.46,60.54,60.84,74.71,128.23,128.97,129.12,136.76$, 155.24, 170.65, 170.8, 173.22. Anal. calcd for $\mathrm{C}_{25} \mathrm{H}_{28} \mathrm{~N}_{2} \mathrm{O}_{6}: \mathrm{C}, 66.36 ; \mathrm{H}, 6.24 ; \mathrm{N}, 6.19$. Found: $\mathrm{C}$, 66.39; H, 6.29; N, 6.11.

Dibutyl 1,3-di-(2,5-dioxo-4,4-diphenylimidazolidin-1-yl)propanoate (5b). Colourless oil. FTIR $\left(v_{\max }, \mathrm{cm}^{-1}\right): 2959,2874,1774,1715,1452,1014,770,697 .{ }^{1} \mathrm{H} \mathrm{NMR}\left(400 \mathrm{MHz}, \mathrm{CDCl}_{3}\right): \delta_{\mathrm{H}}$ 0.89 (t, 3H, J 7.2 Hz, $\left.\mathrm{CH}_{3}\right), 0.92\left(\mathrm{t}, 3 \mathrm{H}, J 7.6 \mathrm{~Hz}, \mathrm{CH}_{3}\right), 1.25-1.40$ (m, 4H, 2CH$), 1.48-1.59$ (m, $\left.4 \mathrm{H}, 2 \mathrm{CH}_{2}\right), 1.90\left(\mathrm{t}, 2 \mathrm{H}, J 7.6 \mathrm{~Hz}, \mathrm{CH}_{2}\right), 2.73\left(\mathrm{t}, 2 \mathrm{H}, J 7.2 \mathrm{~Hz}, \mathrm{CH}_{2}\right), 3.67\left(\mathrm{t}, 2 \mathrm{H}, J 7.6 \mathrm{~Hz}, \mathrm{CH}_{2}\right)$, $3.90\left(\mathrm{t}, 2 \mathrm{H}, J 7.2 \mathrm{~Hz}, \mathrm{CH}_{2}\right), 3.95$ (t, $\left.2 \mathrm{H}, J 6.8 \mathrm{~Hz}, \mathrm{CH}_{2}\right), 4.03$ (t, 2H, J $6.8 \mathrm{~Hz}, \mathrm{CH}_{2}$ ), 7.27-7.29 (m, 4H, Ar), 7.40-7.42 (m, 6H, Ar). $\left.{ }^{13} \mathrm{C} \mathrm{NMR} \mathrm{(100} \mathrm{MHz,} \mathrm{CDCl}_{3}\right): \delta_{\mathrm{C}} 13.69,13.72,19.04,19.08$, 30.46, 30.50, 32.24, 32.61, 35.22, 37.46, 64.43, 64.76, 74.69, 128.21, 128.97, 129.11, 136.76, 155.22, 170.72, 170.87, 173.27. Anal. calcd for $\mathrm{C}_{29} \mathrm{H}_{36} \mathrm{~N}_{2} \mathrm{O}_{6}: \mathrm{C}, 68.48 ; \mathrm{H}, 7.13 ; \mathrm{N}, 5.51$. Found: C, 68.41; H, 7.18; N, 5.49.

Dihexyl 1,3-di-(2,5-dioxo-4,4-diphenylimidazolidin-1-yl)propanoate (5c). Colourless oil. FTIR $\left(v_{\max }, \mathrm{cm}^{-1}\right): 3062,2931,2859,1774,1717,1452,1186,770,696,604,496 .{ }^{1} \mathrm{H}$ NMR (400 $\left.\mathrm{MHz}, \mathrm{CDCl}_{3}\right): \delta_{\mathrm{H}} 0.87-0.89\left(\mathrm{~m}, 6 \mathrm{H}, 2 \mathrm{CH}_{3}\right), 1.26-1.36\left(\mathrm{~m}, 12 \mathrm{H}, 6 \mathrm{CH}_{2}\right), 1.51-1.59\left(\mathrm{~m}, 2 \mathrm{H}, \mathrm{CH}_{2}\right)$, $1.57\left(\mathrm{t}, 2 \mathrm{H}, J 6.8 \mathrm{~Hz}, \mathrm{CH}_{2}\right), 1.91\left(\mathrm{t}, 2 \mathrm{H}, J 8.0 \mathrm{~Hz}, \mathrm{CH}_{2}\right), 2.73\left(\mathrm{t}, 2 \mathrm{H}, J 6.8 \mathrm{~Hz}, \mathrm{CH}_{2}\right), 3.67(\mathrm{t}, 2 \mathrm{H}$, $\left.J 8.0 \mathrm{~Hz}, \mathrm{CH}_{2}\right), 3.91\left(\mathrm{t}, 2 \mathrm{H}, J 6.8 \mathrm{~Hz}, \mathrm{CH}_{2}\right), 3.96\left(\mathrm{t}, 2 \mathrm{H}, J 6.8 \mathrm{~Hz}, \mathrm{CH}_{2}\right), 4.03(\mathrm{t}, 2 \mathrm{H}, J 6.8 \mathrm{~Hz}$, $\mathrm{CH}_{2}$ ), 7.26-7.29 (m, 4H, Ar), 7.40-7.42 (m, 6H, Ar). $\left.{ }^{13} \mathrm{C} \mathrm{NMR} \mathrm{(100} \mathrm{MHz}, \mathrm{CDCl}_{3}\right): \delta_{\mathrm{C}} 13.95$, 13.96, 22.46, 25.42, 25.45, 28.33, 28.38, 31.30, 31.35, 32.16, 32.50, 35.18, 37.42, 64.53, 64.87, 74.57, 128.19, 128.84, 128.97, 133.54, 136.84, 155.11, 170.57, 170.67, 173.07. Anal. calcd for $\mathrm{C}_{33} \mathrm{H}_{44} \mathrm{~N}_{2} \mathrm{O}_{6}$ : C, 70.19; H, 7.85; N, 4.96. Found: C, 70.25; H, 7.91; N, 4.98.

Di-2-Ethylhexyl 1,3-di-(2,5-dioxo-4,4-diphenylimidazolidin-1-yl)propanoate (5d). Colourless oil. FT-IR $\left(v_{\max }, \mathrm{cm}^{-1}\right): 2958,2930,1775,1727,1735,1454,1420,1184,769,696,603 .{ }^{1} \mathrm{H}$ NMR $\left(400 \mathrm{MHz}, \mathrm{CDCl}_{3}\right): \delta_{\mathrm{H}} 0.8-0.88\left(\mathrm{~m}, 12 \mathrm{H}, 4 \mathrm{CH}_{3}\right), 1.20-1.35\left(\mathrm{~m}, 16 \mathrm{H}, 8 \mathrm{CH}_{2}\right), 1.46-1.51(\mathrm{~m}$, $1 \mathrm{H}, \mathrm{CH}), 1.53-1.56(\mathrm{~m}, 1 \mathrm{H}, \mathrm{CH}), 1.91\left(\mathrm{t}, 2 \mathrm{H}, J 8.0 \mathrm{~Hz}, \mathrm{CH}_{2}\right), 2.72\left(\mathrm{t}, 2 \mathrm{H}, J 7.2 \mathrm{~Hz}, \mathrm{CH}_{2}\right), 3.66(\mathrm{t}$, $\left.2 \mathrm{H}, J 8.0 \mathrm{~Hz}, \mathrm{CH}_{2}\right), 3.85-3.91\left(\mathrm{~m}, 4 \mathrm{H}, 2 \mathrm{CH}_{2}\right), 3.92-3.99\left(\mathrm{~m}, 2 \mathrm{H}, \mathrm{CH}_{2}\right), 7.27-7.28$ (m, 4H, Ar), 7.38-7.39 (m, 6H, Ar). ${ }^{13} \mathrm{C} \mathrm{NMR}\left(100 \mathrm{MHz}, \mathrm{CDCl}_{3}\right): \delta_{\mathrm{C}} 10.92,10.94,14.03,14.06,22.92,22.95$, 23.66, 28.81, 28.86, 30.27, 32.20, 32.51, 35.16, 37.49, 38.55, 38.59, 66.89, 67.23, 74.67, 128.20, 128.93, 129.05, 136.81, 155.19, 170.71, 170.87, 173.14. Anal. calcd for $\mathrm{C}_{37} \mathrm{H}_{52} \mathrm{~N}_{2} \mathrm{O}_{6}: \mathrm{C}, 71.58$; H, 8.44; N, 4.51. Found: C, 71.52; H, 8.50; N, 4.55.

Diethyl 2-(2,5-dioxo-4,4-diphenylimidazolidin-1-yl)succinate (6a). White crystal, mp129-131 ${ }^{\circ} \mathrm{C}$. IR $\left(v_{\max }, \mathrm{cm}^{-1}\right): 3232,2983,1777,1740,1715,1429,1287,1182,697 .{ }^{1} \mathrm{H}$ NMR (400 MHz, $\left.\mathrm{CDCl}_{3}\right): \delta_{\mathrm{H}} 1.02\left(\mathrm{t}, 3 \mathrm{H}, J 7.2 \mathrm{~Hz}, \mathrm{CH}_{3}\right), 1.08\left(\mathrm{t}, 3 \mathrm{H}, J 7.2 \mathrm{~Hz}, \mathrm{CH}_{3}\right), 2.98,3.19\left(\mathrm{AMX}, 2 \mathrm{H}, \delta_{\mathrm{A}}=\right.$ $\left.2.98 \mathrm{ppm}, \delta_{\mathrm{M}}=3.19 \mathrm{ppm}, J_{\mathrm{AM}}=16.4 \mathrm{~Hz}, J_{\mathrm{AX}}=9.2 \mathrm{~Hz}, J_{\mathrm{MX}}=6.0 \mathrm{~Hz}, \mathrm{CH}_{2}\right), 3.91-4.03(\mathrm{~m}, 2 \mathrm{H}$, $\left.\mathrm{CH}_{2}\right), 4.06-4.15\left(\mathrm{~m}, 2 \mathrm{H}, \mathrm{CH}_{2}\right), 5.14\left(\mathrm{AMX}, 1 \mathrm{H}, \delta_{\mathrm{X}}=5.14 \mathrm{ppm}, J_{\mathrm{XA}}=9.2 \mathrm{~Hz}, J_{\mathrm{XM}}=6.0 \mathrm{~Hz}, \mathrm{CH}\right)$, 
6.95 (s, 1H, NH), 7.18-7.32 (m, 10H, Ar). $\left.{ }^{13} \mathrm{C} \mathrm{NMR} \mathrm{(100} \mathrm{MHz,} \mathrm{CDCl}_{3}\right): \delta_{\mathrm{C}} 13.87,13.95,33.69$, 49.19, 61.04, 62.36, 70.28, 127.11, 128.66, 128.78, 138.67, 138.90, 155.42, 167.81, 169.79, 172.69. Anal. calcd for $\mathrm{C}_{23} \mathrm{H}_{24} \mathrm{~N}_{2} \mathrm{O}_{6}$ : C, 65.08; H, 5.70; N, 6.60. Found: C, 65.13; H, 5.65; N, 6.69 .

Dipropyl 2-(2,5-dioxo-4,4-diphenylimidazolidin-1-yl)succinate (6b). White Crystal, mp80-82 ${ }^{\circ} \mathrm{C}$. FT-IR $\left(v_{\max }, \mathrm{cm}^{-1}\right): 3324,2965,2937,1773,1721,1449,1423,1268,1184,707,603 .{ }^{1} \mathrm{H}$ NMR (400 MHz, $\left.\mathrm{CDCl}_{3}\right): \delta_{\mathrm{H}} 0.83\left(\mathrm{t}, 3 \mathrm{H}, J 7.2 \mathrm{~Hz}, \mathrm{CH}_{3}\right), 0.85\left(\mathrm{t}, 3 \mathrm{H}, J 7.6 \mathrm{~Hz}, \mathrm{CH}_{3}\right), 1.47-1.6$ $\left(\mathrm{m}, 4 \mathrm{H}, 2 \mathrm{CH}_{2}\right), 3.11,3.32\left(\mathrm{AMX}, 2 \mathrm{H}, \delta_{\mathrm{A}}=3.11 \mathrm{ppm}, \delta_{\mathrm{M}}=3.32 \mathrm{ppm}, J_{\mathrm{AM}}=17.6 \mathrm{~Hz}, J_{\mathrm{AX}}=8.8\right.$ $\left.\mathrm{Hz}, J_{\mathrm{MX}}=6.2 \mathrm{~Hz}, \mathrm{CH}_{2}\right), 3.96\left(\mathrm{t}, 2 \mathrm{H}, J 6.8 \mathrm{~Hz}, \mathrm{CH}_{2}\right), 4.09-4.14\left(\mathrm{~m}, 2 \mathrm{H}, \mathrm{CH}_{2}\right), 5.28\left(\mathrm{AMX}, 1 \mathrm{H}, \delta_{\mathrm{X}}\right.$ $\left.=5.28 \mathrm{ppm}, J_{\mathrm{XA}}=8.8 \mathrm{~Hz}, J_{\mathrm{XM}}=6.2 \mathrm{~Hz}, \mathrm{CH}\right), 7.04(\mathrm{~s}, \mathrm{br}, 1 \mathrm{H}, \mathrm{NH}), 7.29-7.45(\mathrm{~m}, 10 \mathrm{H}, \mathrm{Ar}) .{ }^{13} \mathrm{C}$ NMR $\left(100 \mathrm{MHz}, \mathrm{CDCl}_{3}\right): \delta_{\mathrm{C}} 10.21,10.25,21.70,21.75,33.69,49.21,66.66,67.94,70.30$, 76.75, 77.06, 77.38, 127.10, 127.12, 128.66, 128.77, 128.78, 138.67, 138.94, 155.42, 167.95, 169.89, 172.69. Anal. calcd for $\mathrm{C}_{25} \mathrm{H}_{28} \mathrm{~N}_{2} \mathrm{O}_{6}$ : C, 66.36; H, 6.24; N, 6.19. Found: C, 66.44; H, $6.21 ; \mathrm{N}, 6.13$.

Dibuyl 2-(2,5-dioxo-4,4-diphenylimidazolidin-1-yl)succinate (6c). Colourless oil. FT-IR ( $v_{\max }$, $\left.\mathrm{cm}^{-1}\right): 3325,2967,2935,1781,1730,1422,1273,700,669,603 .{ }^{1} \mathrm{H} \mathrm{NMR}\left(400 \mathrm{MHz}, \mathrm{CDCl}_{3}\right): \delta_{\mathrm{H}}$ $0.86\left(\mathrm{t}, 3 \mathrm{H}, J 7.6 \mathrm{~Hz}, \mathrm{CH}_{3}\right), 0.89$ (t, 3H, J 7.2 Hz, $\left.\mathrm{CH}_{3}\right), 1.21-1.34\left(\mathrm{~m}, 4 \mathrm{H}, 2 \mathrm{CH}_{2}\right), 1.46-1.54(\mathrm{~m}$, $\left.4 \mathrm{H}, 2 \mathrm{CH}_{2}\right), 3.09,3.31\left(\mathrm{AMX}, 2 \mathrm{H}, \delta_{\mathrm{A}}=3.09 \mathrm{ppm}, \delta_{\mathrm{M}}=3.31 \mathrm{ppm}, J_{\mathrm{AM}}=16.8 \mathrm{~Hz}, J_{\mathrm{AX}}=8.6 \mathrm{~Hz}\right.$, $\left.J_{\mathrm{MX}}=6.2 \mathrm{~Hz}, \mathrm{CH}_{2}\right), 4.0\left(\mathrm{t}, 2 \mathrm{H}, J 6.8 \mathrm{~Hz}, \mathrm{CH}_{2}\right), 4.09-4.16\left(\mathrm{~m}, 2 \mathrm{H}, \mathrm{CH}_{2}\right), 5.27\left(\mathrm{AMX}, 1 \mathrm{H}, \delta_{\mathrm{X}}=\right.$ $\left.5.27 \mathrm{ppm}, J_{\mathrm{XA}}=8.6 \mathrm{~Hz}, J_{\mathrm{XM}}=6.2 \mathrm{~Hz}, \mathrm{CH}\right), 7.0(\mathrm{~s}, \mathrm{br}, 1 \mathrm{H}, \mathrm{NH}), 7.29-7.44(\mathrm{~m}, 10 \mathrm{H}, \mathrm{Ar}) .{ }^{13} \mathrm{C}$ NMR $\left(100 \mathrm{MHz}, \mathrm{CDCl}_{3}\right): \delta_{\mathrm{C}} 13.64,13.67,18.89,19.00,30.31,30.39,33.70,49.19,64.98$, 66.24, 70.28, 127.08, 127.11, 128.66, 128.78, 128.79, 138.71, 138.95, 155.39, 167.94, 169.90, 172.64. Anal. calcd for $\mathrm{C}_{27} \mathrm{H}_{32} \mathrm{~N}_{2} \mathrm{O}_{6}$ : C, 67.48; H, 6.71; N, 5.83. Found: C, 67.52; H, 6.75; N, 5.81 .

Dipentyl 2-(2,5-dioxo-4,4-diphenylimidazolidin-1-yl)succinate (6d). White Crystal, mp78-80 ${ }^{\circ} \mathrm{C}$. FT-IR $\left(v_{\max }, \mathrm{cm}^{-1}\right): 3312,2934,2957,1781,1731,1423,1264,1179,978,699 .{ }^{1} \mathrm{H}$ NMR (400 $\left.\mathrm{MHz}, \mathrm{CDCl}_{3}\right): \delta_{\mathrm{H}} 0.76\left(\mathrm{t}, 3 \mathrm{H}, J 7.2 \mathrm{~Hz}, \mathrm{CH}_{3}\right), 0.78\left(\mathrm{t}, 3 \mathrm{H}, J 8.0 \mathrm{~Hz}, \mathrm{CH}_{3}\right), 1.12-1.19(\mathrm{~m}, 8 \mathrm{H}$, $\left.4 \mathrm{CH}_{2}\right), 1.37-1.44\left(\mathrm{~m}, 4 \mathrm{H}, 2 \mathrm{CH}_{2}\right), 2.95,3.19\left(\mathrm{AMX}, 2 \mathrm{H}, \delta_{\mathrm{A}}=2.95 \mathrm{ppm}, \delta_{\mathrm{M}}=3.19 \mathrm{ppm}, J_{\mathrm{AM}}=\right.$ $\left.16.0 \mathrm{~Hz}, J_{\mathrm{AX}}=8.0 \mathrm{~Hz}, J_{\mathrm{MX}}=6.0 \mathrm{~Hz}, \mathrm{CH}_{2}\right), 3.87\left(\mathrm{t}, 2 \mathrm{H}, J 8.0 \mathrm{~Hz}, \mathrm{CH}_{2}\right), 3.97-4.02\left(\mathrm{~m}, 2 \mathrm{H}, \mathrm{CH}_{2}\right)$, $5.15\left(\mathrm{AMX}, 1 \mathrm{H}, \delta_{\mathrm{X}}=5.15 \mathrm{ppm}, J_{\mathrm{XA}}=8.0 \mathrm{~Hz}, J_{\mathrm{XM}}=6.0 \mathrm{~Hz}, \mathrm{CH}\right), 7.18-7.33(\mathrm{~m}, 10 \mathrm{H}, \mathrm{Ar}), 7.41$ (s, 1H, NH). ${ }^{13} \mathrm{C}$ NMR $\left(100 \mathrm{MHz}, \mathrm{CDCl}_{3}\right): \delta_{\mathrm{C}} 13.88,13.95,22.18,22.24,27.11,27.88,27.96$, 28.05, 33.73, 49.14, 65.23, 66.48, 70.27, 127.06, 128.57, 128.74, 138.77, 138.98, 155.64, 167.94, 169.89, 172.66. Anal. calcd for $\mathrm{C}_{29} \mathrm{H}_{36} \mathrm{~N}_{2} \mathrm{O}_{6}$ : C, 68.48; H, 7.13; N, 5.51. Found: C, 68.52; H, $7.10 ; \mathrm{N}, 5.57$.

Dioctyl 2-(2,5-dioxo-4,4-diphenylimidazolidin-1-yl)succinate (6e). Colourless oil. FT-IR $\left(v_{\max }, \mathrm{cm}^{-1}\right): 3354,2928,1780,1721,1429,1266,1177,757,698 .{ }^{1} \mathrm{H} \mathrm{NMR}\left(400 \mathrm{MHz}, \mathrm{CDCl}_{3}\right)$ : $\delta_{\mathrm{H}} 0.78-0.82\left(\mathrm{~m}, 6 \mathrm{H}, 2 \mathrm{CH}_{3}\right), 1.14-1.16\left(\mathrm{~m}, 20 \mathrm{H}, 10 \mathrm{CH}_{2}\right), 1.41-1.42\left(\mathrm{~m}, 4 \mathrm{H}, 2 \mathrm{CH}_{2}\right), 2.97,3.21$ $\left(\mathrm{AMX}, 2 \mathrm{H}, \delta_{\mathrm{A}}=2.97 \mathrm{ppm}, \delta_{\mathrm{M}}=3.21 \mathrm{ppm}, J_{\mathrm{AM}}=16.4 \mathrm{~Hz}, J_{\mathrm{AX}}=8.4 \mathrm{~Hz}, J_{\mathrm{MX}}=6.4 \mathrm{~Hz}, \mathrm{CH}_{2}\right)$, $3.91\left(\mathrm{t}, 2 \mathrm{H}, J 6.8 \mathrm{~Hz}, \mathrm{CH}_{2}\right), 3.93-4.07\left(\mathrm{~m}, 2 \mathrm{H}, \mathrm{CH}_{2}\right), 5.16\left(\mathrm{AMX}, 1 \mathrm{H}, \delta_{\mathrm{X}}=5.16 \mathrm{ppm}, J_{\mathrm{XA}}=8.4\right.$ $\left.\mathrm{Hz}, J_{\mathrm{XM}}=6.4 \mathrm{~Hz}, \mathrm{CH}\right), 6.70(\mathrm{~s}, \mathrm{br}, 1 \mathrm{H}, \mathrm{NH}), 7.18-7.33(\mathrm{~m}, 10 \mathrm{H}, \mathrm{Ar}) .{ }^{13} \mathrm{C} \mathrm{NMR}(100 \mathrm{MHz}$, 
$\left.\mathrm{CDCl}_{3}\right): \delta_{\mathrm{C}} 13.88,13.95,14.11,22.18,22.24,26.62,26.76,28.29,28.38,29.12,29.17,31.78$, $33.70,49.19,65.27,66.53,70.27,76.73,127.11,127.27,128.64,128.77,138.72,138.94,155.32$, 167.91, 169.88, 172.61. Anal. calcd for $\mathrm{C}_{35} \mathrm{H}_{48} \mathrm{~N}_{2} \mathrm{O}_{6}$ : C, 70.92; H, 8.16; N, 4.73. Found: C, $70.87 ; \mathrm{H}, 8.18 ; \mathrm{N}, 4.80$.

Didecyl 2-(2,5-dioxo-4,4-diphenylimidazolidin-1-yl)succinate (6f). Colourless oil. FT-IR $\left(v_{\max }, \mathrm{cm}^{-1}\right): 3255,2292,2854,1780,1740,1710,1423,1270,1178,757,698 .{ }^{1} \mathrm{H}$ NMR $(400$ $\left.\mathrm{MHz}, \mathrm{CDCl}_{3}\right): \delta_{\mathrm{H}} 0.78-0.82\left(\mathrm{~m}, 6 \mathrm{H}, \mathrm{CH}_{3}\right), 1.13-1.24\left(\mathrm{~m}, 28 \mathrm{H}, 14 \mathrm{CH}_{2}\right), 1.38-1.44\left(\mathrm{~m}, 4 \mathrm{H}, 2 \mathrm{CH}_{2}\right)$, 2.94, $3.19\left(\mathrm{AMX}, 2 \mathrm{H}, \delta_{\mathrm{A}}=2.94 \mathrm{ppm}, \delta_{\mathrm{M}}=3.19 \mathrm{ppm}, J_{\mathrm{AM}}=16.4 \mathrm{~Hz}, J_{\mathrm{AX}}=8.4 \mathrm{~Hz}, J_{\mathrm{MX}}=7.4\right.$ $\mathrm{Hz}, \mathrm{CH}_{2}$ ), 3.51 (t, 2H, J $\left.6.8 \mathrm{~Hz}, \mathrm{CH}_{2}\right), 3.85-4.05\left(\mathrm{~m}, 2 \mathrm{H}, \mathrm{CH}_{2}\right), 5.15\left(\mathrm{AMX}, 1 \mathrm{H}, \delta_{\mathrm{X}}=5.15 \mathrm{ppm}\right.$, $\left.J_{\mathrm{XA}}=8.4 \mathrm{~Hz}, J_{\mathrm{XM}}=7.4 \mathrm{~Hz}, \mathrm{CH}\right), 7.25-7.31(\mathrm{~m}, 11 \mathrm{H}, \mathrm{NH}, \mathrm{Ar}) .{ }^{13} \mathrm{C} \mathrm{NMR}\left(100 \mathrm{MHz}, \mathrm{CDCl}_{3}\right): \delta_{\mathrm{C}}$ $13.88,13.94,14.13,22.69,25.61,25.77,28.29,28.38,29.17,29.22,29.32,29.45,29.52,29.55$, 29.64, 31.90, 32.75, 33.73, 49.14, 62.97, 65.29, 66.50, 70.27, 127.09, 128.56, 128.73, 139.00, 155.53, 167.94, 169.89, 172.69. Anal. calcd for $\mathrm{C}_{39} \mathrm{H}_{56} \mathrm{~N}_{2} \mathrm{O}_{6}: \mathrm{C}, 72.19 ; \mathrm{H}, 8.70 ; \mathrm{N}, 4.32$. Found: C, 72.22; H, 8.76; N, 4.43.

\section{Acknowledgements}

The authors are thankful to University of Mohaghegh Ardabili and Tehran University Councils for their partial support to the work.

\section{References}

1. Avendaño Lopez, C.; Gonzales Trigo, G. Adv. Heterocycl. Chem. 1985, 38, 177-228. http://dx.doi.org/10.1016/S0065-2725(08)60920-4

2. Scholl, S.; Koch, A.; Henning, D.; Kempter, G.; Kleinpeter, E. Struct. Chem. 1999, 10, $355-$ 366. http://dx.doi.org/10.1023/a:1022091411018

3. Knabe, J.; Baldauf, J.; Ahlhem, A. Pharmazie 1997, 52, 912-919. PMid:9480456

4. Rodgers, T. R.; Lamontagne, M. P.; Markovac, A.; Ash, A. B. J. Med. Chem. 1977, 20, 591594.

http://dx.doi.org/10.1021/jm00214a031

5. Wessels, F. L.; Schwan, T. J.; Pong, S. F. J. Pharm. Sci. 1980, 69, 1102-1104. PMid:6106054

6. El-Barbarly, A. A.; Khodair, A. I.; Pedersen, E. B.; Nielson, C. J. Med. Chem. 1994, 37, 7377. http://dx.doi.org/10.1021/jm00027a009 
7. Comber, R. N.; Reynolds, R. C.; Friedrich, J. D.; Manguikian, R. A.; BuckheitJr, R. W.; Truss, J. W.; Shannon, W. M.; Secrist III, J. A. J. Med. Chem. 1992, 35, 3567-3572. PMid:1404236

8. Goodnow Jr, R. A.; Huby, N. J. S.; Kong, N.; McDermott, L. A.; Moliterni, J. A.; Zhang, Z. US. 2008. (US 7371869 B2 ed).

9. Ware, E. Chem. Rev. 1950, 46, 403-470. http://dx.doi.org/10.1021/cr60145a001

10. Mahmoodi, N. O.; Khodaee, Z. Arkivoc 2007, (iii), 29-36.

11. Zhang, D.; Xing, X.; Cuny, G. D. J. Org. Chem. 2006, 71, 1750-1753. http://dx.doi.org/10.1021/jo052474s

12. Brown, M. L.; Brown, G. B.; Brouillette, W. J. J. Med. Chem. 1997, 40, 602-607. http://dx.doi.org/10.1021/jm960692v

13. Cloyd, M. W.; Lynn, W. S.; Ramsey, K.; Baron, S. Virology 1989, 173, 581-590. http://dx.doi.org/10.1016/0042-6822(89)90569-2

14. Alizadeh, A.; Sheikhi, E. Tetrahedron Lett. 2007, 48, 4887-4890. http://dx.doi.org/10.1016/j.tetlet.2007.05.061

15. Mclean, M. J.; Macdonald, R. L. J. Pharmacol. Exp. Ther. 1983, 227, 779-789.

16. Maggini, M.; Prato, M.; Ranelli, M.; Scorrano, G. Tetrahedron Lett. 1992, 33, 6537-6540. http://dx.doi.org/10.1016/S0040-4039(00)79036-8

17. Corriu, R. J. P.; Perz, R. Tetrahedron Lett. 1985, 26, 1311-1314. http://dx.doi.org/10.1016/S0040-4039(00)94879-2

18. Bredereck, H.; Gompper, R.; Herlinger, H.; Wotiun, E. Eur. J. Inorg. Chem. 1960, 93, 24232429. http://dx.doi.org/10.1002/cber.19600931042

19. Reitz, A.; Verlander, M.; Goodman, M. Tetrahedron Lett. 1982, 23, 751-752. http://dx.doi.org/10.1016/S0040-4039(00)86938-5

20. Mariella, R. P.; Jonauskas, R. J. Org. Chem. 1958, 23, 923-923. http://dx.doi.org/10.1021/jo01100a620

21. Ruiz, E.; Rodriguez, H.; Coro, J.; Salfran, E.; Suarez, M.; Martinez-Alvarez, R.; Martin, N. Ultrason. Sonochem. 2011, 18, 32-36. http://dx.doi.org/10.1016/j.ultsonch.2010.04.009

22. Mirza-Aghayan, M.; Etemad-Moghadam, G.; Zaparucha, A.; Berlan, J.; Loupy, A.; Koenig, M. Tetrahedron: Asymmetry 1995, 6, 2643-2646. http://dx.doi.org/10.1016/0975-4166(95)00346-Q

23. Said, K.; Moussaoui, Y.; Kammoun, M.; Salem, R. B. Ultrason. Sonochem. 2011, 18, 23-27. http://dx.doi.org/10.1016/j.ultsonch.2010.04.007

24. Sinha, A. K.; Sharma, A.; Joshi, B. P. Tetrahedron 2007, 63, 960-965. http://dx.doi.org/10.1016/j.tet.2006.11.023

25. Kumar, V.; Sharma, A.; Sharma, M.; Sharma, U. K.; Sinha, A.K. Tetrahedron 2007, 63, 9718-9723. 
http://dx.doi.org/10.1016/j.tet.2007.07.018

26. Imanzadeh, G. H.; Zare, A.; Khalafinezhad, A.; Hasaninejad, A.; Mosavi Zare, A. R.; Parhami, A. J. Iran. Chem. Soc. 2007, 4, 467-475. http://dx.doi.org/10.1007/BF03247234

27. Imanzadeh, G. H.; Kazemi, F.; Zamanloo, M.; Mansoori, Y. Ultrason. Sonochem. 2013, 20, 722-728.

http://dx.doi.org/10.1016/j.ultsonch.2012.09.003

28. Moshtael, N.; Safari, J. Ultrason. Sonochem. 2011, 18, 640-643.

http://dx.doi.org/10.1016/j.ultsonch.2010.09.001

29. Aki, S. N. V. K.; Brennecke, J. F.; Samanta, A. Chem. Commun. 2001, 413-414. http://dx.doi.org/10.1039/b008039j

30. Vogel's Textbook of Practical Organic Chemistry, A. $4^{\text {th }}$ ed. Longman Press: London, 1978. 